\title{
Nonlinear piezoelectric vibration energy harvesting from a portal frame with two-to-one internal resonance
}

\author{
Rodrigo T. Rocha $\cdot$ Jose M. Balthazar $\cdot$ Angelo M. Tusset $\cdot$ \\ Vinicius Piccirillo $\cdot$ Jorge L. P. Felix
}

Received: 24 March 2016/Accepted: 1 February 2017 / Published online: 22 February 2017

(C) Springer Science+Business Media Dordrecht 2017

\begin{abstract}
In the few decades, the study of electromechanical systems which are capable to extract energy from an operating system in the environment has been of most importance. In this work, we present the extraction of energy from a simple portal frame structure excited by a harmonic force, where the energy harvesting is computed by using of a nonlinear piezoelectric material. The dynamical response of the system is examined, when there is $2: 1$ internal resonance between the symmetric and the sway mode, resulting the saturation phenomenon and vibration
\end{abstract}

R. T. Rocha $(\varangle) \cdot$ A. M. Tusset · V. Piccirillo UTFPR - Federal University of Technology - Parana, Ponta Grossa, PR 83016-210, Brazil

e-mail: digao.rocha@gmail.com

A. M. Tusset

e-mail: tusset@utfpr.edu.br

V. Piccirillo

e-mail: piccirillo@utfpr.edu.br

J. M. Balthazar

Faculty of Mechanical Engineering, UNESP - Sao Paulo

State University, CP 473, Bauru, SP 17033-360, Brazil

e-mail: jmbaltha@ita.br

J. M. Balthazar

ITA - Aeronautics Technological Institute,

Sao Jose dos Campos, SP 12228-900, Brazil

J. L. P. Felix

UFFS - Federal University of Fronteira Sul, Cerro Largo,

RS 97900-000, Brazil

e-mail: jorge.felix@uffs.edu.br energy transfer between the symmetric (vertical) mode and the horizontal (sway) mode. An evaluation of the energy available for harvesting, in each of the considered modes, is computed.

Keywords Energy harvesting $\cdot$ Nonlinear dynamics $\cdot$ Portal frame structure $\cdot$ Saturation phenomenon

\section{Introduction}

Recently, there has been much interest in the concepts of electro-mechanical systems that are able to scavenge, or harvest energy from their operating environment. As the kinetic energy is a source of energy easily found in the environment, devices that converts kinetic energy into electrical energy have been widely studied, and special attention has been devoted to devices that use piezoelectric elements as a means of energy transduction.

Many researchers have recently explored this kind of energy harvesting based on piezoelectric materials. Piezoceramics can be used as a lot of devices, for example, as piezomagnetoelastic structure and harvest energy from an ambient vibration [1-6]. A vast and important study of piezoelectric energy harvesting can be found at [7-13]. These authors explored the re-use of wasted vibration energy in the environment, which is a very important subject, nowadays, to some applications, including renewable energy. 
It is known in the literature about energy harvesting that there is a certain nonlinearity in the material related to the $d_{31}$ coefficient. Specifically, there is a nonlinear relation between the strain and the electric field of the piezoelectric material [12, 13].

The nonlinearity of the piezoelectric material was identified experimentally by [14]. Due to the significant contribution of the nonlinearity of the piezoelectric material, an approximation was proposed by [15], in order to model a nonlinear piezoelectric relation. Recently, a complete revision of these properties was presented by [16]. Many authors introduced the nonlinear piezoelectric coupling on their works and showed that the energy harvesting can be more or less efficient; in special we mention the works [17-20]. The analysis of the nonlinear piezoelectric factor will be discussed, in this work.

Some kind of structures of two-degrees-of-freedom are specially coupled with an associated quadratic nonlinearity. Moreover, these structures may possess 2:1 internal resonance, and when they are subjected to an external resonance due to an external excitation, saturation phenomenon is presented.

Saturation phenomenon is when a system partiallytransfer vibration energy of the excited mode to another one. The phenomenon has been proposed as a control device and a means for transferring vibration energy from one region of a system to another, where it may be easier for harvesting. Thus, we will analze the possible use of these features, using a two-degreesof-freedom simple portal frame, as support structure.

The occurrence of saturation phenomenon was presented in a problem of a ship motion because of its nonlinear coupling of pitch and roll modes [21]. The investigation of a nonlinear control method based on the saturation phenomenon in systems coupled with quadratic nonlinearities, was treated in more details by [22-26]. The implementation of an active saturation control was proposed in [27-30] and studied by [31-36]. In addition, saturation phenomenon was used to suppress steady-state vibrations of a system by connecting it to a second-order controller using quadratic position coupling terms [34]. We also remark that a number of authors have shown that the saturation-based on nonlinear control method for a nonlinear problem requires an adaptive frequency-tuning mechanism, because the frequency of the nonlinear system changes with the amplitude. Hence, the controller will become out of tune with the system to be controlled [37-40].
In earlier work, a model of an energy harvester based on a simple portal frame structure was presented [17]. The system was considered a non-ideal system (NIS), due to a full interaction of the structure motions, with the energy source, a DC motor with limited power supply. The nonlinearities presented in the piezoelectric material were considered in the piezoelectric coupling mathematical model. In addition, the system was found to be a bi-stable Duffing oscillator presenting chaotic behaviour. Moreover, in [18] a portal frame structure was controlled using a Nonlinear Energy Sink (NES) [42], and after addition of piezoelectric elements, energy harvesting was considered. The results showed that the harvested energy with a NES passive controller was improved, since the system was forced to a periodic orbit.

Afterwards, the analysis of the saturation phenomenon with the piezoelectric material coupled to a two-degrees-of-freedom portal frame structure was analyzed by $[19,20]$.

There is a great necessity of study the saturation phenomenon in the structure and the nonlinearity presented in the piezoelectric material. With the saturation phenomenon the structure may behave like periodically or chaotically. A vast study of the vibration modes was performed in order to find out the best periodic configuration in order to harvest energy. The nonlinearities presented in piezoelectric material can be the energy harvesting more or less efficient. It will be important because it will be possible to simulate a most real problem of energy harvesting.

The results obtained, in this paper, showed numerical simulations of these phenomena and energy harvesting, using a nonlinear piezoelectric material as a means of energy transduction coupled to a column of a two-degrees-of-freedom portal frame structure. Extensive analysis of the system was carry out in order to evaluate the energy available for harvesting in each of the considered modes. Hence, this paper was carefully organized as follows:

Section 1 is an introduction of the topic of saturation phenomenon and piezoelectric material discussed in this paper,

Section 2 is the modelling of the system in question. The equations of motion were developed by the method of energy of Lagrange. After that, the analysis of the vibration energy transferred was performed in order to see the saturation phenomenon as studied by [19-36], Section 3 discussed about the 
frequency of the external force, because it is an important factor of the saturation.

Section 4 was carried out an overview of the piezoelectric coupling considering some cases of the use of the frequency. In a first moment, in Sect. 4.1, the case of sole linear piezoelectric coefficient was considered. In the following, in Sect. 4.2, it was considered the nonlinear piezoelectric coefficient mixing the variation of three parameters discussed that are the frequency, linear piezoelectric coefficient and nonlinear piezoelectric coefficient,

Section 5 was performed a vast study of the amplitude of the external force to the case of a fixed value of the sole linear piezoelectric coefficient. Analysis of stability and range of the parameter versus initial velocity condition was carefully made out in order to summarize the stability of the system and to obtain the best stable value of the initial velocity condition. Following with the new initial condition, in Sect. 5.1, the influence of the sole linear piezoelectric coupling versus amplitude was performed, obtaining the best value of the linear coupling. Hence, in Sect. 5.2, the influence of the nonlinear piezoelectric coefficient to the linear piezoelectric coefficient versus amplitude was considered. We showed stability, behaviour and energy harvesting analysis comparing every result obtained here to Sect. 5.1. As it is being considered the nonlinear piezoelectric coefficient we have the most real possible result of energy harvesting, this section becomes the most significant study of Sect. 5 ,

Finally, Sect. 6 we have the conclusions of this work, describing all the conclusions, benefits and disadvantages of each section.

\section{The engineering problem: modelling and derivation of the governing equations of motion, energy transfer process between the two vibrating modes and the vibrating system}

Considering the portal frame of the Fig. 1, consisting of two columns clamped in their bases with height $h$ and a horizontal beam pinned to the columns at both ends with length $L$. Both columns and beam have flexural stiffness $E I$. The mass at mid-span of the beam is $M$. We consider that $m$ represent the masses of the columns. The structure is modelled as a lumped mass system with two-degrees-of-freedom. The coordinate
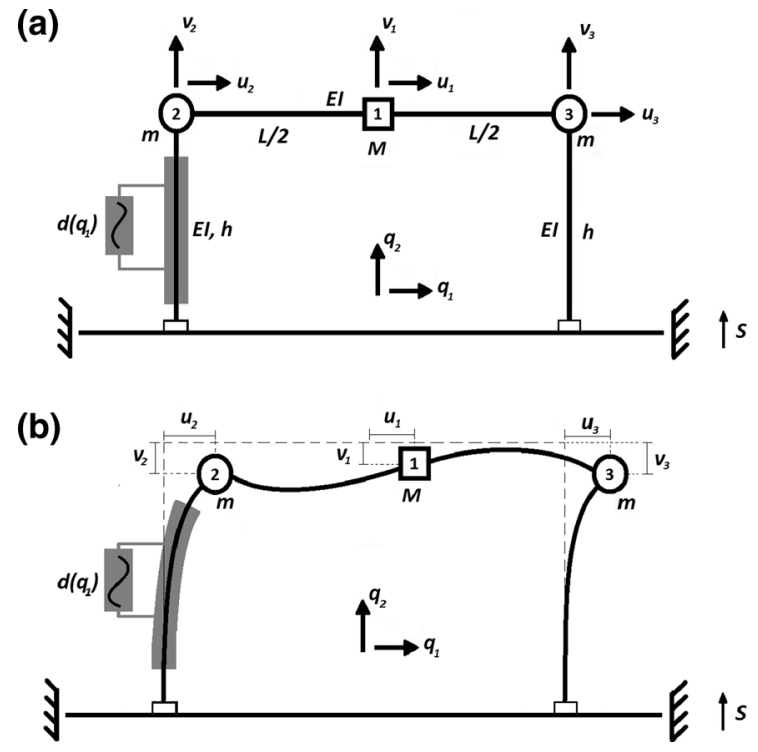

Fig. 1 The physical model of a simple portal frame. a Static position. b Dynamic position

$q_{1}$ is related to the horizontal displacement (sway mode), with natural frequency $\omega_{1}$, and $q_{2}$ is related to the vertical displacement (first symmetrical mode), with natural frequency $\omega_{2}$. The generalized coordinates $q_{i}$ are the displacements of the mass at the midspan of the beam $M$.

The linear stiffness of the columns and the beam can be evaluated by a Rayleigh-Ritz procedure using cubic trial functions. Geometric nonlinearity is introduced by considering the shortening due to bending of the columns and of the beam. We can approximate the length of the columns and beam, respectively, as the equations below.

$$
\begin{array}{ll}
u=\frac{3}{h^{3}}\left(\frac{h x^{2}}{2}-\frac{x^{3}}{6}\right), & 0 \leq x \leq h \\
v=\frac{12}{L^{3}}\left(\frac{x^{3}}{3}-\frac{L^{2} x}{4}\right), & 0 \leq x \leq \frac{L}{2}
\end{array}
$$

Nodal displacements, shown in Fig. 1, are

$$
\begin{aligned}
& u_{1}=q_{1} \quad u_{2}=u_{1}+\frac{B}{4 v_{1}^{2}} \quad u_{3}=u_{1}-\frac{B}{4} v_{1}^{2} \\
& v_{1}=q_{2} \quad v_{2}=-\frac{A}{2} u_{1}^{2} \quad v_{3}=-\frac{A}{2} u_{1}^{2}
\end{aligned}
$$

The constants $A$ and $B$ and the stiffness of the beam and columns, respectively $k_{b}$ and $k_{c}$ are calculated by the Rayleigh method, whose integers are below. 


$$
\begin{aligned}
& k_{c}=E I \int_{0}^{h}\left(u^{\prime \prime}\right)^{2} d x \quad k_{b}=2 E I \int_{0}^{L / 2}\left(v^{\prime \prime}\right)^{2} d x \\
& A=\int_{0}^{h}\left(u^{\prime}\right)^{2} d x \quad B=2 \int_{0}^{L / 2}\left(v^{\prime}\right)^{2} d x
\end{aligned}
$$

As the length of the beam is approximated by half of its value, the calculus of its stiffness and constant $\mathrm{B}$ need to be multiplied by 2 . Hence, we have $A=6 / 5 h$ and $B=24 / 5 L$. The stiffness of the beam is $k_{b}=$ $48 E I / L^{3}$ and the stiffness of the columns is $k_{c}=3 E I / h^{3}$. Our generalized coordinates are the displacements of the mass at the mid-span of the beam "M". Using displacements of Eq. (1), the kinetic energy is

$$
T=\frac{1}{2} M\left(\dot{u}_{1}^{2}+\dot{v}_{1}^{2}\right)+\frac{1}{2} m\left(2 \dot{u}_{1}^{2}\right)
$$

Introducing the generalized coordinates $q_{1}$ and $q_{2}$ in Eq. (2), the kinetic energy becomes

$$
T=\frac{1}{2} M\left(\dot{q}_{1}^{2}+\dot{q}_{2}^{2}\right)+\frac{1}{2} m\left(2 \dot{q}_{1}^{2}\right)
$$

The system has a coupled electrical circuit at the column which is excited by an internal voltage (backemf) proportional to the mechanical velocity. The circuit consists of a resistor $R$, the charge $Q$, a capacitor $C_{p}$ and the dimensionless piezoelectric contribution is given by $d\left(q_{1}\right)=\theta\left(1+\Theta\left|q_{1}\right|\right)$ [15-20], where $\theta$ is the linear piezoelectric coefficient and $\Theta$ is the nonlinear piezoelectric coefficient.

The potential energy of the system is given by the strain energy of the structure, the work of the weight of the masses of the beam and columns and by electrical potential of the circuit with the contribution of the piezoelectric and the capacitor, resulting

$$
\begin{aligned}
U= & \frac{1}{2} k_{c}\left(u_{2}^{2}+u_{3}^{2}\right)+\frac{1}{2} k_{b}\left(v_{1}-\frac{v_{2}+v_{3}}{2}\right)^{2} \\
& +\cdots m g\left(v_{2}+v_{3}\right)+M g v_{1}-\frac{d\left(q_{1}\right)}{C_{p}} Q\left(u_{2}+v_{2}\right) \\
& +\frac{1}{2} \frac{Q^{2}}{C_{p}}
\end{aligned}
$$

Substituting the Eq. (1) in (4), in terms of the general coordinates $q_{1}, q_{2}$ and $Q$, we have

$$
\begin{aligned}
U= & \left(k_{c}-m g A\right) q_{1}^{2}+\frac{1}{2} k_{b}\left(q_{2}+A q_{2} q_{1}^{2}\right)+M g q_{2} \\
& -\cdots \frac{d\left(q_{1}\right)}{C_{p}} Q\left(q_{1}+\frac{B}{4} q_{2}^{2}\right)+\frac{1}{2} \frac{Q^{2}}{C_{p}}
\end{aligned}
$$

Now, we consider energy dissipation of the system, comprising the structural damping defined by a Rayleigh function and the resistor of the electrical circuit. Then it follows

$D=\frac{1}{2} c_{1} \dot{q}_{1}^{2}+\frac{1}{2} c_{2} \dot{q}_{1}^{2}+\frac{1}{2} R \dot{Q}^{2}$

The mechanical system is excited by a harmonic force which has amplitude $F_{0}$ and external frequency $\omega_{n}$, namely

$$
S=F_{0} \cos \omega_{n} t
$$

The Lagrangian function is defined by Eq. (8). Substituting Eqs. (3) and (5) into (8), we have the Lagrangian of Eq. (9).

$$
\begin{aligned}
L(q, \dot{q}, t)=T-U \\
\begin{aligned}
L= & \frac{1}{2} M\left(\dot{q}_{1}^{2}+\dot{q}_{2}^{2}\right)+\frac{1}{2} m\left(2 \dot{q}_{1}^{2}\right)-\left(k_{c}-m g A\right) q_{1}^{2} \\
& -\cdots \frac{1}{2} k_{b}\left(q_{2}+A q_{2} q_{1}^{2}\right)-M g q_{2} \\
& +\cdots \frac{d\left(q_{1}\right)}{C_{p}} Q\left(q_{1}+\frac{B}{4} q_{2}^{2}\right)-\frac{1}{2} \frac{Q^{2}}{C_{p}}
\end{aligned}
\end{aligned}
$$

Now, using Euler-Lagrange, that is Eq. (10), we have the governing equations of motion of the system that are Eqs. (11), (12) and (13).

$$
\begin{aligned}
& \frac{d}{d t}\left(\frac{\partial L}{\partial \dot{q}_{i}}\right)-\frac{\partial L}{\partial q_{i}}+\frac{\partial D}{\partial \dot{q}_{i}}=F_{e x t} \quad i=1,2,3 \\
& (2 m+M) \ddot{q}_{1}+2\left(k_{c}-m g A\right) q_{1}+k_{b} A q_{1} q_{2}+c_{1} \dot{q}_{1} \\
& =\cdots \frac{d\left(q_{1}\right)}{C_{p}} Q \\
& M \ddot{q_{2}}+k_{b} q_{2}+c_{2} \dot{q}_{2}=F_{0} \cos \omega_{n} t-M g-\frac{A k_{b}}{2} q_{1}^{2}+ \\
& \ldots \frac{d\left(q_{1}\right)}{C_{p}} \frac{B}{2} Q q_{2}
\end{aligned}
$$




$$
R \dot{Q}-\theta\left(1+\Theta\left|q_{1}\right|\right) \frac{1}{C_{p}}\left(q_{1}+\frac{B}{4} q_{2}^{2}\right)+\frac{Q}{C_{p}}=0
$$

Next, a dimensionless process was carried out, resulting the equations of the system as follows

$$
\begin{aligned}
x_{1}^{\prime \prime} & +\mu_{1} x_{1}^{\prime}+x_{1}+\alpha_{1} x_{1} x_{2}=\theta\left(1+\Theta\left|x_{1}\right|\right) \delta_{1} V \\
x_{2}^{\prime \prime} & +\mu_{2} x_{2}^{\prime}+\omega_{2}^{2} x_{2}+\alpha_{2} x_{1}^{2}+G_{0}=E_{0} \cos \Omega \tau \\
& +\cdots \theta\left(1+\Theta\left|x_{1}\right|\right) \delta_{2} V x_{2} \\
& +\cdots\left(1+\Theta\left|x_{1}\right|\right)\left(\delta_{3} x_{1}+\delta_{4} x_{2}^{2}\right)+\delta_{3} V=0
\end{aligned}
$$

where

$$
\begin{aligned}
& x_{1}=\frac{q_{1}}{h} \quad x_{2}=\frac{q_{2}}{L} \quad V=\frac{Q}{q_{0}} \quad \tau=\omega_{1} t \\
& \omega_{1}=\sqrt{\frac{2\left(k_{c}-m g A\right)}{2 m+M} \quad \bar{d}\left(x_{1}\right)=\frac{h}{q_{0}} d\left(q_{1}\right) \quad G_{0}=\frac{g}{\omega_{1}^{2} L}} \\
& \mu_{1}=\frac{c_{1}}{(2 m+M) \omega_{1}} \quad \mu_{2}=\frac{c_{2}}{M \omega_{1}} \quad \omega_{2}=\frac{1}{\omega_{1}} \sqrt{\frac{k_{b}}{M}} \\
& \alpha_{1}=\frac{A k_{b} L}{(2 m+M) \omega_{1}^{2}} \quad \alpha_{2}=\frac{A k_{b} h^{2}}{2 M \omega_{1}^{2} L} \quad \Omega=\frac{\omega_{n}}{\omega_{1}} \\
& \delta_{1}=\frac{q_{0}^{2}}{\omega_{1}^{2} h^{2}(2 m+M) C_{p}} \quad \delta_{2}=\frac{B q_{0}^{2}}{2 M \omega_{1}^{2} h C_{p}} \\
& \delta_{3}=\frac{1}{R C_{p} \omega_{1}} \quad \delta_{4}=\frac{B L^{2}}{4 R C_{p} \omega_{1} h} \quad E_{0}=\frac{F_{0}}{M \omega_{1}^{2} L}
\end{aligned}
$$

The external frequency $\Omega$ was set near resonance with the first symmetrical mode. Frequency $\omega_{2}$ is also set twice the frequency of the sway mode as $2 \omega_{1}=\omega_{2}$, in order to have modal coupling in the nonlinear adopted model. The conditions below are necessary to saturation phenomenon occurs [19-36, 40, 41], which will be seen in the next section.

$$
\begin{aligned}
& \omega_{2}=2 \omega_{1}+\sigma_{1} \\
& \omega_{2}=\omega_{n}+\sigma_{2} \\
& \mu_{1}, \mu_{2} \ll 1 \\
& \sigma_{1}, \sigma_{2} \ll 1
\end{aligned}
$$

where $\sigma$ is a detuning factor, and $\mu_{1}$ and $\mu_{2}$ are dampings.
The calculation of the harvested power for the portal frame system are given by the Eqs. (17) and (18) that describe the dimensional and dimensionless harvested power, respectively.

$P=R \dot{Q}^{2}$

$P=R_{0} V^{\prime 2}$

where $R_{0}=R\left(\omega_{1} q_{0}\right)^{2}$.

The average power of the system can be calculated by the Eq. (19), as in [15-20].

$$
P_{\text {avg }}=\frac{1}{T} \int_{0}^{T} P(\tau) d \tau
$$

In the following, the numerical simulation results were performed using the software MATLAB, considering all the parameters of the Table 1

On the other hand, the parameters related to external excitation frequency $\omega_{n}$, linear piezoelectric coefficient $\theta$ and the nonlinear piezoelectric coefficient $\Theta$ will be varied, accordingly.

The most interesting of saturation phenomenon is the transferring of the vibration energy of modes, so that one of the modes has higher amplitude at steady state. In this case, as the first symmetrical mode was excited, the vibration energy will be transferred from this mode to the sway mode. This energy transfer is a nonlinear phenomenon, which may eventually occur

Table 1 Adopted system parameters

\begin{tabular}{lll}
\hline Parameters & Values & Means \\
\hline$g\left(\mathrm{~m} / \mathrm{s}^{2}\right)$ & 9.81 & Gravity acceleration \\
$M(\mathrm{~kg})$ & 2.00 & Beam mass \\
$m(\mathrm{~kg})$ & 0.50 & Column mass \\
$c_{1}(\mathrm{Ns} / \mathrm{m})$ & 1.55 & Column damping \\
$c_{2}(\mathrm{Ns} / \mathrm{m})$ & 3.14 & Beam damping \\
$E I(\mathrm{~nm})^{2}$ & 128 & Linear stiffness \\
$L(\mathrm{~m})$ & 0.52 & Beam length \\
$h(\mathrm{~m})$ & 0.36 & Column length \\
$F_{0}(\mathrm{~N})$ & 40 & External excitation amplitude \\
$R(\mathrm{k} \Omega)$ & 100 & Piezoelectric resistance \\
$C_{p}(\mu \mathrm{F})$ & 1 & Piezoelectric capacitance \\
$\omega_{n}(\mathrm{rad} / \mathrm{s})$ & Varied & External excitation frequency \\
$\theta$ & Varied & Linear piezoelectric coefficient \\
$\Theta$ & Varied & Nonlinear piezoelectric coefficient
\end{tabular}


when a system possess a 2:1 internal resonance. Saturation phenomenon is called when the process of energy transfer ends and the vibration energy keeps changed itself [21-26]. Hence, the first step is to verify the energy transferring of the symmetric mode to the sway mode.

Note that the total mechanical energy of the sway mode, and the symmetric mode and the total energy of the electrical system are given, respectively, by the Eqs. (21), (22) and (23), according to [43, 44].

$$
\begin{aligned}
& E_{x_{1}}=\left(k_{c}-m g A\right) x_{1}^{2}+\frac{1}{2}(M+2 m) x_{1}^{\prime 2} \\
& E_{x_{2}}=\frac{1}{2} k_{b} x_{2}^{2}+M g x_{2}+\frac{1}{2} M x_{2}^{\prime 2} \\
& E_{V}=\frac{1}{2 C_{p}} V^{2}
\end{aligned}
$$

The total energy of the electromechanical system is given by the sum of Eqs. (21), (22) and (23), i.e., $E_{\text {total }}=E_{x_{1}}+E_{x_{2}}+E_{V}$.

To analyze the energy transfer between the two vibrating modes and the electrical part, the algebraic relations of the energy percentage, are considered and defined by the relations (24).

$$
\begin{aligned}
& E_{\% x_{1}}=\frac{E_{x_{1}}}{E_{\text {total }}} \\
& E_{\% x_{2}}=\frac{E_{x_{2}}}{E_{\text {total }}} \\
& E_{\% V}=\frac{E_{V}}{E_{\text {total }}}
\end{aligned}
$$

Through the numerical values of Table 1, neglecting the use of the piezoelectric material and considering a small initial condition to the sway mode, we will vary the frequency of the external force in order to see the commented phenomena. The following Fig. 2a-f show an analysis of the energy transfer and the appearance of the saturation phenomena.

The adopted portal frame model was analyzed firstly, assuming $\omega_{n}=100 \mathrm{rad} / \mathrm{s}$ whose frequency value does not occur the energy transfer. Figure $2 \mathrm{a}, \mathrm{d}$ show the mechanical energy percentage and we see that the energy transfer starts to occur at the beginning of the simulation, however, after a long time the energy of the sway mode decays to approximately zero while the mechanical energy of the symmetric mode goes to approximately $100 \%$ of the system's energy.
Assuming now that the frequency of $\omega_{2}=\omega_{n}=148 \mathrm{rad} / \mathrm{s}$, which is the condition to saturation phenomenon occurs. Figure $2 b$, e show the mechanical energy of the two modes. The energy transfer phenomenon occurred at transient state. However, differently of the Fig. 2a, d, at steady state, the energy of the first symmetric mode was partially transferred to the sway mode, i.e., the saturation phenomenon occurred.

Next, it was coupled the piezoelectric material to analyze the energy transfer among the two modes and the charge, considering just its linear part with coefficient $\theta=0.3$. Figure $2 \mathrm{c}$, f show the mechanical energy percentage among the three coordinates, and, in comparison to Fig. 2b, e, the sway mode has a little less energy, as expected, because it was transferred to the piezoelectric device.

In the next sections, we analyzed the influence of the external frequency in order to verify the amount of harvested energy and the range of the external frequency, which occurs the energy transfer and saturation phenomena. In addition, the linear piezoelectric coefficient, nonlinear piezoelectric coefficient, and the influence of the amplitude of the external force were analyzed in order to verify the amount of energy harvested.

Therefore, the next section shows some analysis the influence of the external force frequency.

\section{Frequency of the external force}

A careful look at the governing equations of motion [Eqs. (14), (15) and (16)] we may see that the system has a limited number of parameters to study. However, some parameters are important to improve the energy harvesting of the considered system. The study of the influence of these parameters is the most important feature of this work.

The frequency of the external force is a parameter, which has a great influence on the energy harvesting from the system, because of saturation and energy transfer phenomena. When the vibrating system is set with 2:1 internal resonance, there will be a range of values that occurs both phenomena and, consequently, it will be possible to harvest energy from the piezoelectric material coupled to the column of the portal frame. Thereby, a parametrical analysis of the frequency of the external force related to the average 


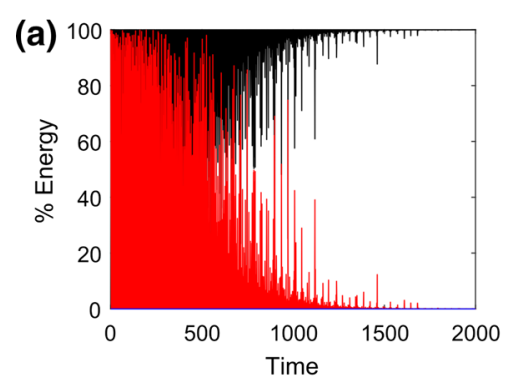

(d)

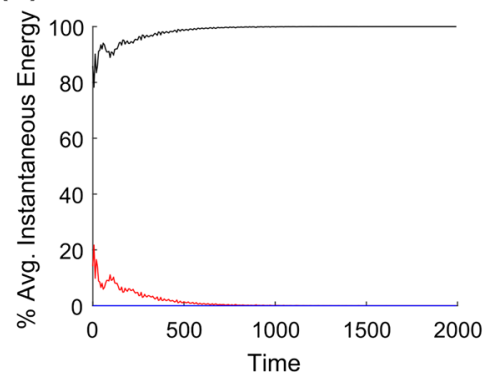

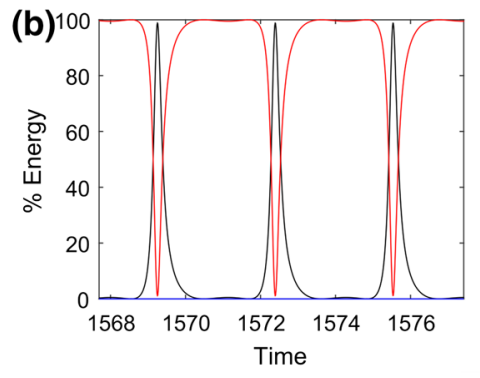

(e)

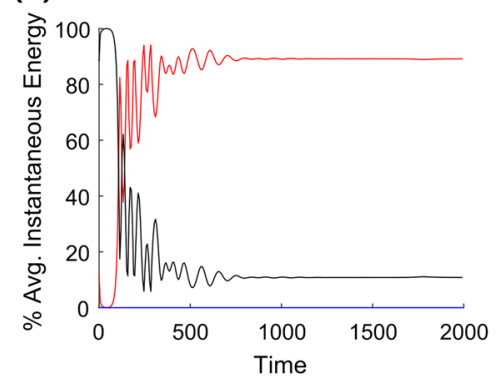

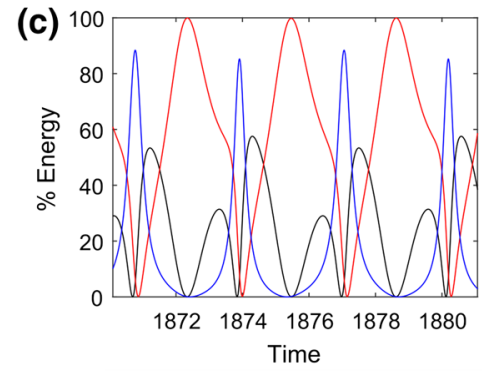

(f)

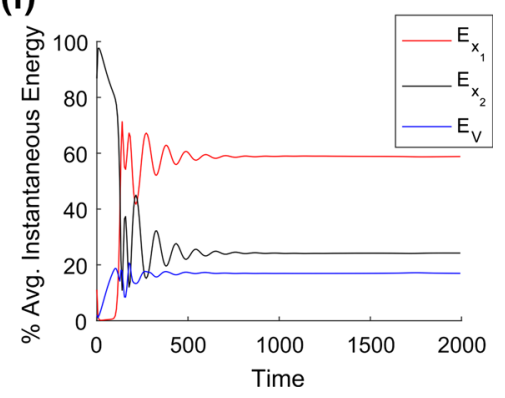

Fig. 2 Energy percentage of the sway mode (red line), symmetric mode (black line) and electrical energy (blue line). a Full time with $\omega_{n}=100 \mathrm{rad} / \mathrm{s}$. b Steady state with $\omega_{n}=148 \mathrm{rad} / \mathrm{s}$. c Steady state considering linear piezoelectric coefficient $\theta=0.3$ and $\omega_{n}=148 \mathrm{rad} / \mathrm{s}$; average instantaneous

power is carried out, using the parameters of the Table 1 and adopting linear piezoelectric coefficient $\theta=0.3$ and neglecting the nonlinear contribution.

Figure 3 shows the analysis of the frequency related to de average power. As expected, there is energy harvesting when the external force frequency is in a range where saturation phenomenon occurs. This range goes from, approximately, 144 to $151.2 \mathrm{rad} / \mathrm{s}$, as shown in Fig. 3a. Analyzing Fig. 3b, we verify that this dimensional range represents the dimensionless range, which goes from, approximately 1.946 to 2.044 . As the condition to saturation phenomenon happens is $\omega_{2}=\Omega+\sigma$, in Fig. 3b we can see the detuning factor actuating at this condition, and conclude that there is an internal resonance $2: 1$ as $\omega_{2}=2 \omega_{1}$. In addition, the maximum harvested power is obtained when frequency is $\Omega=2.041$, that is, at steady state, 38.96, approximately.

In order to study the global dynamic of the system, a bifurcation diagram is built for the external force frequency. Figure 4a shows a bifurcation diagram of the sway mode, and note that, for the same range of the frequency values (1.946-2.044) in Fig. 3b, the saturation occurs. Besides, it is possible to observe a energy percentage of the sway mode (red line), symmetric mode (black line) and electrical energy (blue line). d With $\omega_{n}=100 \mathrm{rad} / \mathrm{s}$. e With $\omega_{n}=148 \mathrm{rad} / \mathrm{s}$. f Considering linear piezoelectric coefficient $\theta=0.3$ and $\omega_{n}=148 \mathrm{rad} / \mathrm{s}$. (Color figure online)
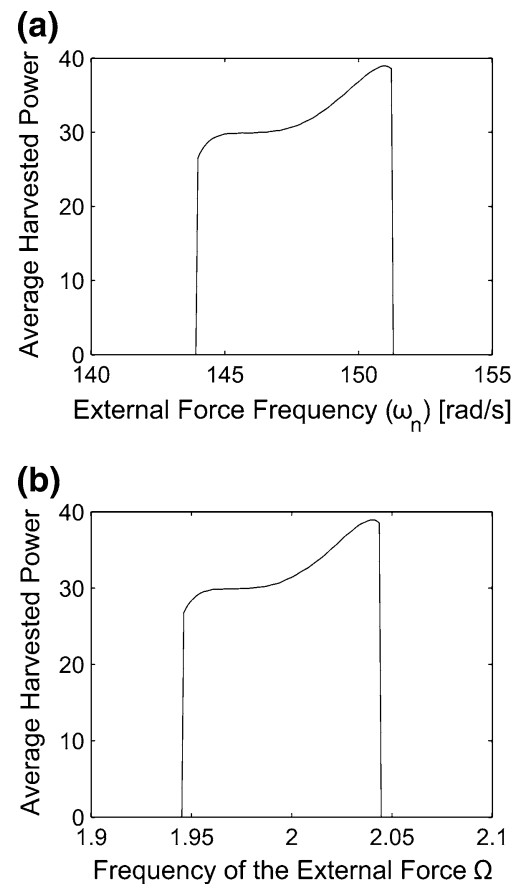

Fig. 3 Frequency of the external force versus average harvested power. a Dimensional frequency. b Dimensionless frequency 
changed behaviour of the sway mode, i.e., when saturation phenomenon takes place, the portal frame system becomes period-2. Figure $4 \mathrm{~b}$ shows a bifurcation diagram of the first symmetric mode. We observe that, in the same range of the frequency values (red area 1), its behaviour also becomes period-2.

As the range of the frequency to have the saturation phenomenon is known, the next section will show the contribution of the piezoelectric coefficient, in order to see the amount of harvested energy.

\section{Piezoelectric coupling}

The piezoelectric device, as commented before, was coupled to one of the columns of the portal frame. It will be used as a means of energy transduction through the vibration of the column, because of saturation phenomenon. However, the piezoelectric material possesses different values of piezoelectric constants. In this section, these constants will be analyzed in dimensionless form to obtain its influence on amount of harvested power by varying it.
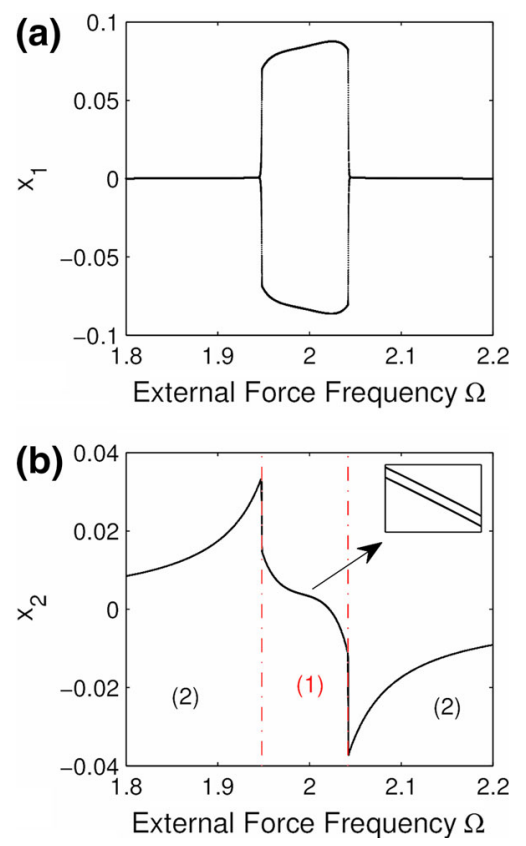

Fig. 4 Bifurcation diagram of the coordinates related to the varying of the external force frequency. a Sway mode. b Symmetric mode
The material is considered as a nonlinear device, considering its relations as $\bar{d}\left(x_{1}\right)=\theta\left(1+\Theta\left|x_{1}\right|\right)$, where $\theta$ is the linear coefficient and $\Theta$ is the nonlinear coefficient, as showed in modelling section. In the following, some analysis of the linear and nonlinear piezoelectric coefficients were discussed.

\subsection{Linear piezoelectric coupling}

Starting in this subsection, the case of the sole linear piezoelectric coefficient is carried out.

Firstly, saturation phenomenon will not be considered in order to compare the amount of power when there is saturation. Then, setting the external force out of resonance, for example $\omega_{n}=100 \mathrm{rad} / \mathrm{s}$ ( $\Omega=1.1315$ ), the linear piezoelectric coupling is $\theta=$ 0.3 and the nonlinear $\Theta=0$ and considering the other parameters of the Table 1, numerical simulations were performed.

Figure 5a shows the time histories of the two modes, and it is possible to see that there is no energy transfer between the two modes. Figure $5 \mathrm{~b}$ shows the almost motionless amplitude of the sway mode. Hence, it will has a little amount of harvested power, due to this almost motionless movement. We can see, too, the periods of the two modes without saturation phenomenon. Figure 6 a shows a superposition of the Poincare map (the red dot) and the phase plane for the sway mode and we may observe that this mode has period-1. Figure $6 \mathrm{~b}$ shows a superposition of the Poincare map (the red dot) and the phase plane for the symmetric mode and we observe that this mode has period-1 too. That is interesting to the maintaining of the energy harvesting because the system is periodic. Figure $7 \mathrm{a}$ shows the time history of the harvested power. Because of the almost motionless movement of the sway mode, we verified a low harvested power, remembering that is due to the fact that the piezoelectric ceramic bar is coupled to the columns of the portal frame. Figure $7 \mathrm{~b}$ shows the average power in the red line at steady state that is approximately only $7.7 \times 10^{-7}$.

Next, saturation is considered setting the external frequency as $\omega_{2}=\omega_{n}=148 \mathrm{rad} / \mathrm{s}(\Omega=2.0)$ (the symmetrical mode natural frequency), which is one of the conditions of the phenomenon. As discussed before, partial vibration energy will be transferred to the column, improving the energy harvesting. 

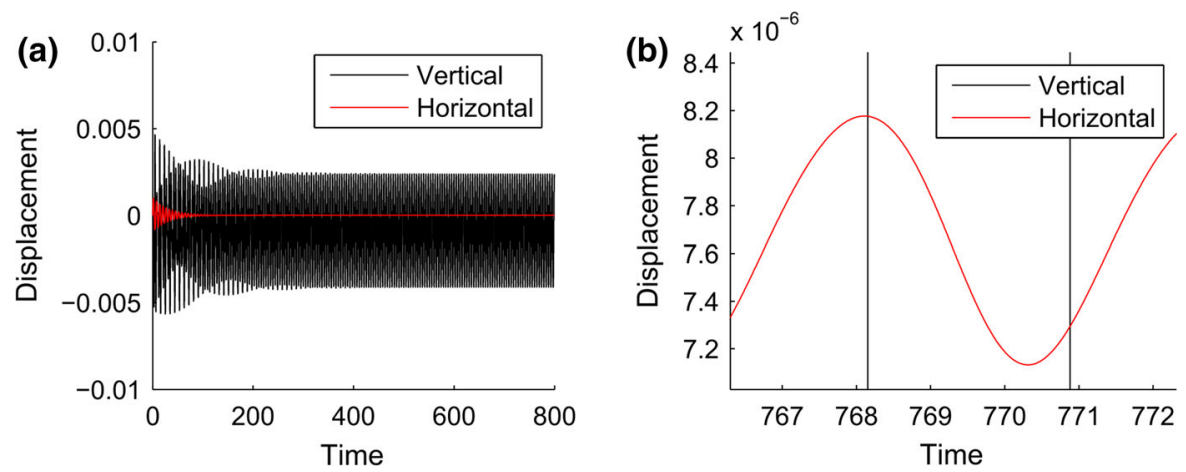

Fig. 5 a Time histories of the two modes. b Approximation of the sway modes curve, $\omega_{n}=100 \mathrm{rad} / \mathrm{s}, \theta=0.3, \Theta=0$
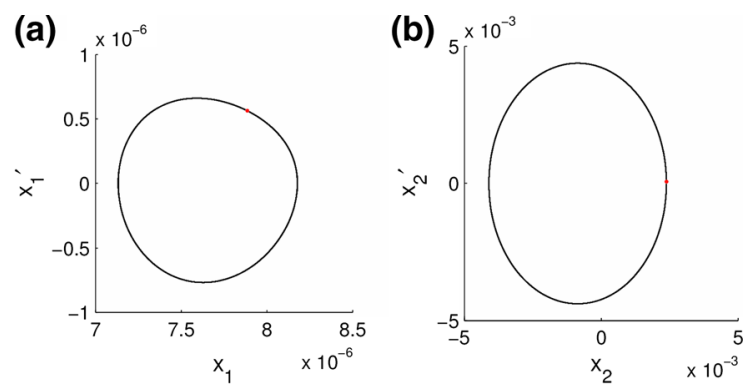

Fig. 6 a Poincare map (red dot) and phase plane for the sway mode at steady state. b Poincare map (red dot) and phase plane for the symmetric mode at steady state, $\omega_{n}=100 \mathrm{rad} / \mathrm{s}$, $\theta=0.3, \Theta=0$. (Color figure online)

Figure 8a shows the saturation phenomenon and energy transfer from the first symmetric mode to the sway mode. Figure 9a, b show the Poincare maps of the two modes of the system. Figure 9a indicates that the sway mode with saturation has period-2 and Fig. $9 \mathrm{~b}$ shows that the symmetric mode is period-2 too, at steady state. Thus, energy harvesting is possible. In Fig. $8 \mathrm{~b}$ we observe that there is a lot more harvested power than in Fig. 7 where saturation does not actuate. The red line in Fig. $8 \mathrm{~b}$ shows the average power at steady state to be approximately 31.37. We can see a great improvement of energy harvesting in the system from approximately $7.7 \times$ $10^{-7}$ to 31.37 harvested power, but less energy than in the case of $(\Omega=2.041)$, which the average harvested power is 38.96 .

As linear piezoelectric coefficient was presented, in the next topic will be discussed the influence of the nonlinear piezoelectric coefficient in the behaviour of the vibrating system and in the energy harvesting.

\subsection{Nonlinear piezoelectric coupling}

The nonlinear piezoelectric coupling has relevant contributions to the system, but it depends on the value of the linear piezoelectric coupling. This coupling makes the system comes close to a real moviment of the system coupled to the piezoelectric material, so that we can simulate a real problem. Looking at the relation of piezoelectric coefficient, the nonlinear
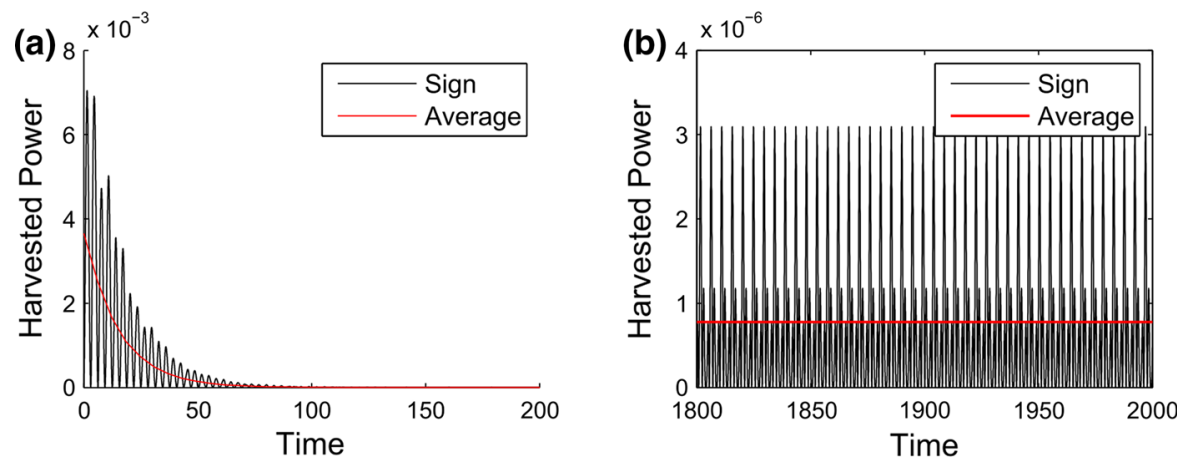

Fig. 7 Time history of the power harvested in signs (black line) and average (red line). a Transient state. b Steady state, $\omega_{n}=100$ rad/ s, $\theta=0.3, \Theta=0$. (Color figure online) 
Fig. 8 a Time histories of the two modes. b Time history of the power harvested, $\omega_{n}=148 \mathrm{rad} / \mathrm{s}$, $\theta=0.3, \Theta=0$
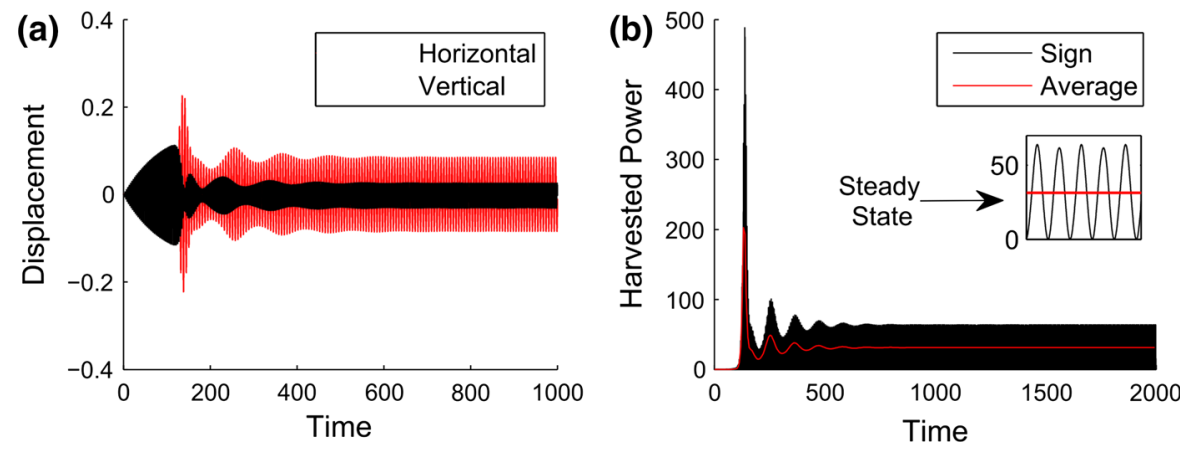

(a)
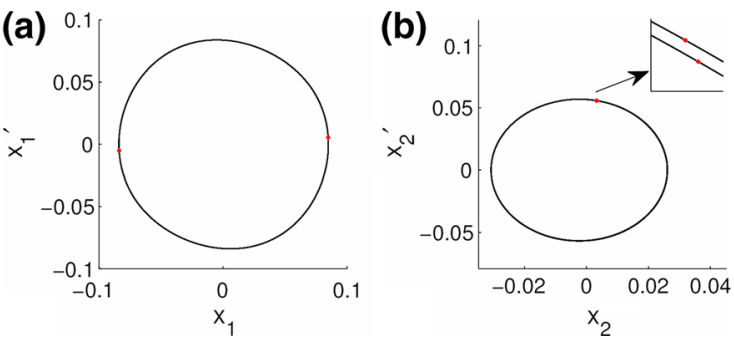

Fig. 9 a Poincare map (red dots) and phase plane for the sway mode at steady state. b Poincare map (red dots) and phase plane for the symmetric mode at steady state, $\omega_{n}=148 \mathrm{rad} / \mathrm{s}$, $\theta=0.3, \Theta=0$. (Color figure online)

coefficient is the angular coefficient of strain curve adjustment of the piezoelectric material. It goes from zero (0) to one (1). To access what happens to the system with the nonlinear coefficient, an analysis of its two parameters in relation to the harvested power was carried out.

First, a parametrical analysis of the linear piezoelectric coefficient neglecting the nonlinear part versus power was performed seeking the maximum and minimum amounts of power. Figure 10a shows the parametrical analysis of the linear piezoelectric coefficient with $\Theta=0$. The value of the linear coefficient, which gives the maximum average power, is $\theta=0.423$.

In order to see what happens to the behaviour of the system varying this parameter, a bifurcation diagram was built. Figure 10b shows a bifurcation diagram of the linear piezoelectric coefficient related to the sway mode with $\Theta=0$. The behaviour of the sway mode from $\theta \approx 0$ to $\theta \approx 0.446$ is period-2. After that, the systems movement continue to be period, but tends to get unstable near $\theta=0.454$. Figure $10 \mathrm{c}$ shows a bifurcation diagram of the linear piezoelectric coefficient related to the symmetric mode with $\Theta=0$. The same periodic behaviour continues and also period-2 to the same range of values of the linear piezoelectric coefficient.

Next, a parametrical analysis of the nonlinear piezoelectric coefficient related to the average harvested power at steady state was validated, considering the value of maximum power of the linear coefficient, which is $\theta=0.423$. Figure 11a displays this analysis keeping the linear piezoelectric coefficients value when the average power is maximum $(\theta=0.423$. It shows that the maximum value of the average power corresponds to the nonlinear piezoelectric coefficient $\Theta=0$. Higher the value of the nonlinear coefficient until $\Theta=1$, lower will be the average harvested power. A bifurcation diagram to the nonlinear piezoelectric coefficient related to the sway mode and symmetric mode is shown in Fig. 11b, c, respectively. They show that the system continue to be period-2.

Now, we analyzed the influence of the nonlinear piezoelectric coefficient in the system through time histories of the displacements and Poincar maps. Considering the found values of the piezoelectric coefficients, that are $\theta=0.423$ and $\Theta=1$, we showed the harvested power with saturation phenomenon and how the system behaves in the next figures.

In the case of the sole linear piezoelectric coupling with maximum average power value $(\theta=0.423$ and $\Theta=0$ ). Figure $12 \mathrm{a}$ shows the time history of the two modes and we can observe the system behave like the system of Fig. 8. It has the energy transfer in the beginning and get the steady state after that. Figure $12 \mathrm{~b}$ shows the harvested power with transient and 
(a)

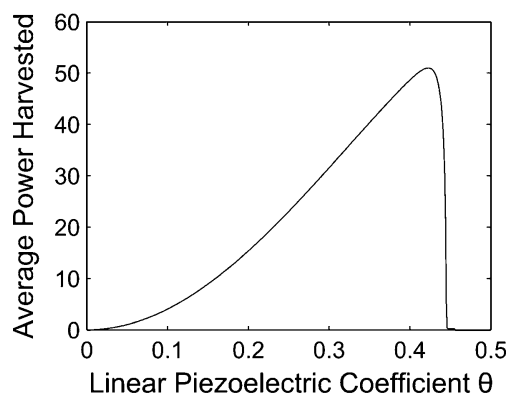

(b)

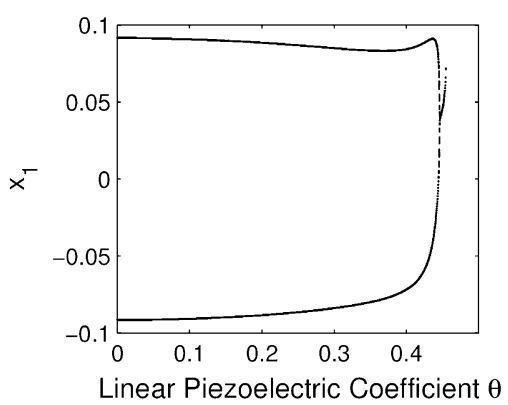

(c)

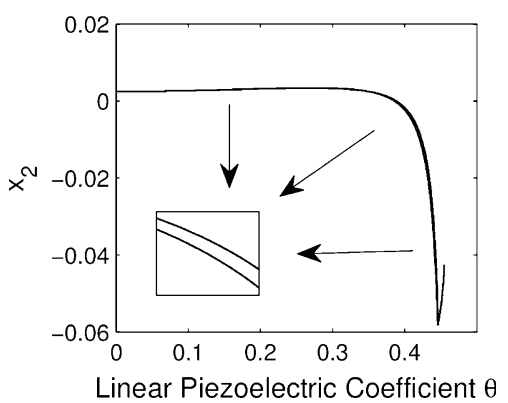

Fig. 10 Linear piezoelectric coefficient analysis. a Parameter versus average harvested power. b Bifurcation diagram related to the sway mode. c Bifurcation diagram related to the symmetric mode

(a)

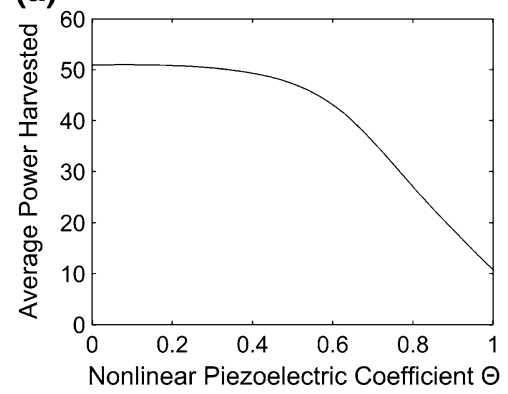

(b)

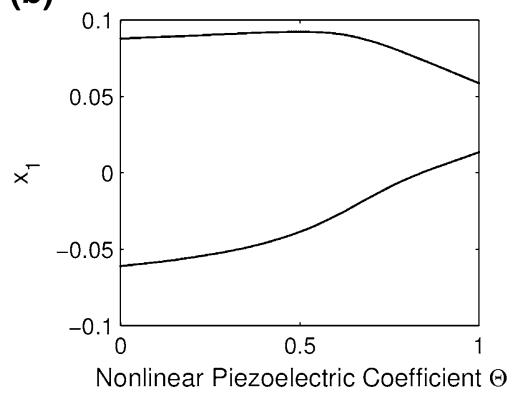

(c)

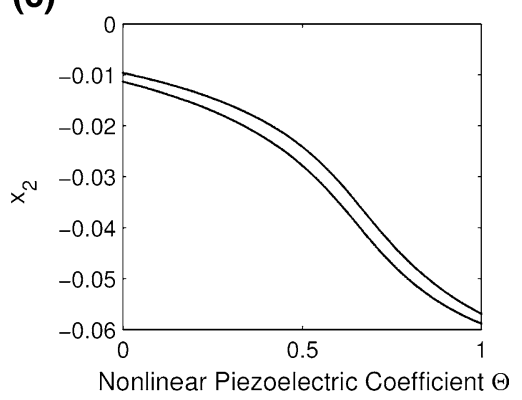

Fig. 11 Nonlinear piezoelectric coefficient analysis. a Parameter versus average harvested power. b Bifurcation diagram related to the sway mode. $\mathbf{c}$ Bifurcation diagram related to the symmetric mode

steady state (black sign) and the average harvested power (red line). The red line shows that the average power at steady state is 50.80, approximately. The Poincare maps of Fig. 13a, b show that the system keeps to be period-2.

Next, we will consider the contribution of the nonlinear piezoelectric coefficient on the system behaviour, using the value $\Theta=1$. Figure 14a shows how saturation behaves with the nonlinear piezoelectric coefficient $\Theta=1$. This should be compared to Fig. 12a.

Figure 15a, b show the Poincare maps of the system, and we see that the behaviour continues to be period-2 in the two modes as predicted in bifurcation diagrams in Fig. 11b, c.

Figure $14 \mathrm{~b}$ shows the harvested power with transient and steady state. We can see that there are less harvested power in comparison to Fig. 12b. The red line shows the average harvested power of the system, and the value now is approximately 10.78 , lower than the linear case (50.80).
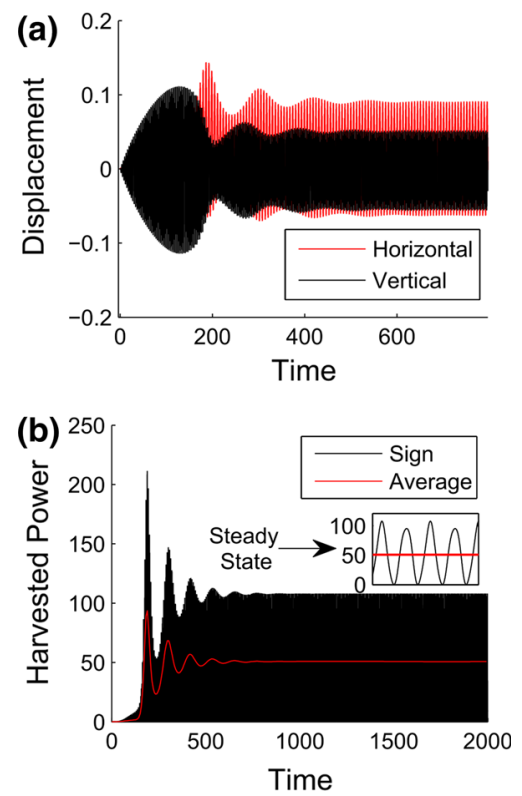

Fig. 12 Time histories of the case of the sole linear piezoelectric coefficient. a Displacements of the two modes. b Harvested power, $\omega_{n}=148 \mathrm{rad} / \mathrm{s}, \theta=0.423, \Theta=0$ 

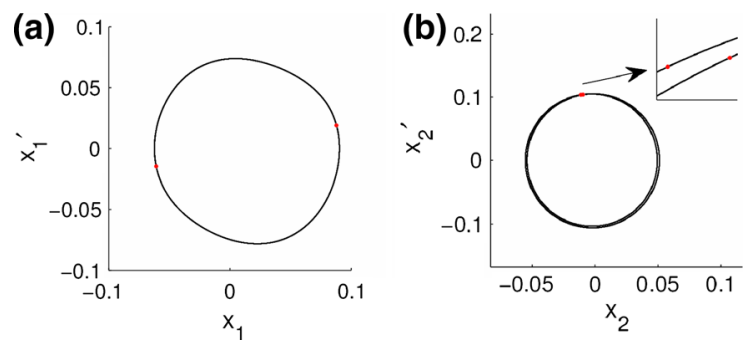

Fig. 13 Poincare map (red dots) and phase planes (black line) of the case of the sole linear piezoelectric coefficient. a Related to the sway mode at steady state. b Related to the symmetric mode at steady state, $\omega_{n}=148 \mathrm{rad} / \mathrm{s}, \theta=0.423, \Theta=0$. (Color figure online)
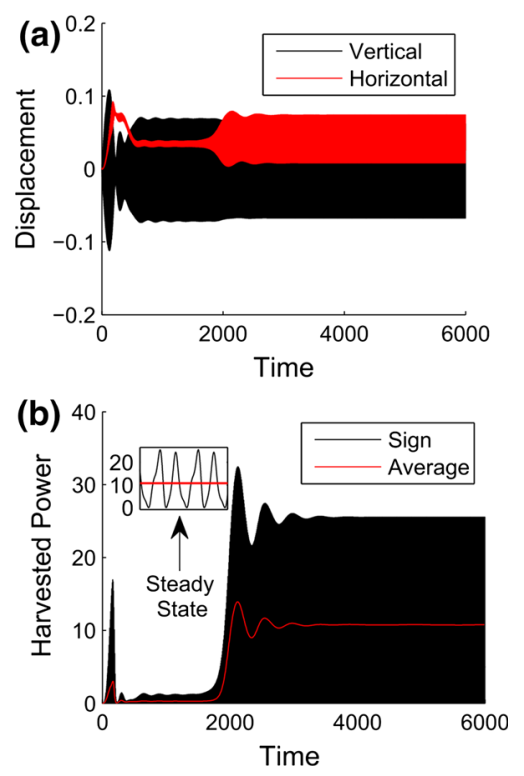

Fig. 14 Time histories of the case of the sole linear piezoelectric coefficient. a Displacements of the two modes. $\mathbf{b}$ Harvested power, $\omega_{n}=148 \mathrm{rad} / \mathrm{s}, \theta=0.423, \Theta=1$
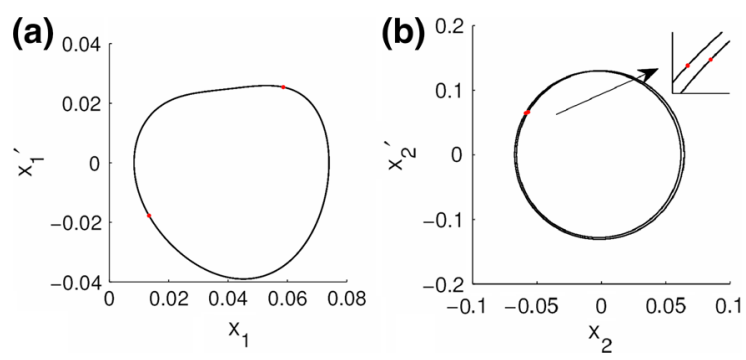

Fig. 15 Poincare map (red dots) and phase planes (black line) of the case of the sole linear piezoelectric coefficient. a Related to the sway mode at steady state. b Related to the symmetric mode at steady state., $\omega_{n}=148 \mathrm{rad} / \mathrm{s}, \theta=0.423, \Theta=1$. (Color figure online)
The contribution of the nonlinear piezoelectric coefficient will depend on the value of the linear piezoelectric coefficient. It can be positive or negative. A good way to see the influence of the nonlinear piezoelectric coefficient on the harvested power is analyzing the results of Fig. 16. With the nonlinear piezoelectric coefficient, we can observe how the system will behave similar the real and having a good improve on the harvested power. Figure 16 allows some conclusions about the studied influence of the nonlinear piezoelectric parameters on the harvested power. We can see that in the acceptable interval of the linear piezoelectric coefficient, that is $0 \leq \theta \leq 0.408$, power has a little gain increasing the nonlinear piezoelectric parameter up to $\Theta=1$. When $\theta>0.409$, power decreases with the nonlinear parameter up to $\Theta=1$. Table 2 shows a brief explanation of the Fig. 16.

Following of the previous analysis of the piezoelectric parameters, Fig. 17 show the influence of varying the parameters in $20 \%$ around $\theta=$ 0.3 and $\Theta=1$, to see the sensibility of the parameters. The average power changes very slowly, then the system does not change suddenly its harvested power, then we can predict how much gain of power the system will have depending on the piezoelectric parameters.

With all the analysis of the piezoelectric coefficients, frequencies and energy transfers, in the next section, will be discussed an important issue to the energy harvesting, that is the amplitude of the external force.
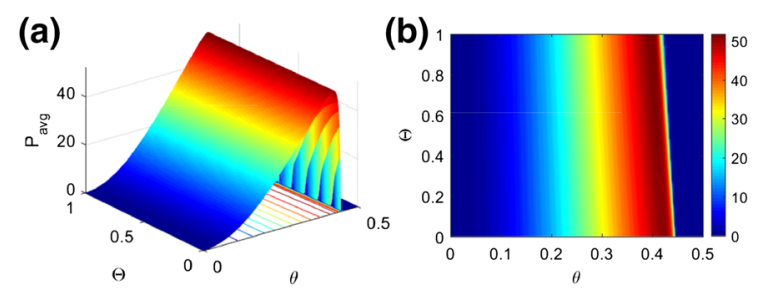

Fig. 16 Analysis of the nonlinear piezoelectric coefficient versus linear coefficient versus average power; a surface, b coloured contour of the surface. (Color figure online) 
Table 2 Brief of the Fig. 16

\begin{tabular}{lllll}
\hline $\mathrm{N}^{\circ}$ & $\theta$ & $\Theta$ & Average power & Brief explanation \\
\hline 1 & 0.401 & 0 & 48.613 & Best gain of power \\
2 & 0.401 & 1 & 52.162 & \\
3 & 0.408 & 0 & 49.547 & Less gain than before \\
4 & 0.408 & 1 & 49.882 & \\
5 & 0.409 & 0 & 49.677 & Loss of power \\
6 & 0.409 & 1 & 48.953 & \\
\hline
\end{tabular}
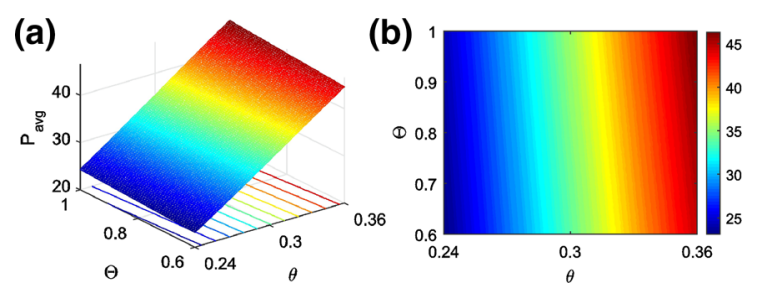

Fig. 17 Analysis of the nonlinear piezoelectric coefficient versus linear coefficient versus average power in an error of approximately 20\%; a surface, b coloured contour of the surface. (Color figure online)

\section{Influence of the amplitude of the external force on the energy harvesting}

The amplitude of the external force is also, of course, a parameter which has a foremost influence on the energy harvesting of the system. The higher the value of the force, the higher will be the energy harvesting. The next simulations were used parameters of the Table 1, except for the force which will be varied. Adopting linear piezoelectric coefficient $\theta=0.3$, nonlinear piezoelectric coefficient $\Theta=0$ and the external force frequency as $\omega_{n}=148 \mathrm{rad} / \mathrm{s}$ $(\Omega=2.0)$.

First, it is important to study the behaviour and stability of the vibrating system related to the increasing of the amplitude of the external force. However, the initial conditions has a great influence of the system. According to some values of the initial conditions, it will have a few limited values of the amplitude to be considered. Then, Fig. 18a shows a surface which varied the initial velocity conditions of the sway mode with the amplitude related to the maximum displacement. The analysis through the results of the surface represent the final amplitude of the displacement at steady state of each parameter relation, i.e., each point represents the same attractor. The peaks of the surface means the steady state response tends to infinite, i.e., it can cause some damage depending on the structure's size due to high amplitudes of displacement. Therefore, these peaks are considered as a unstable behaviour of the system. Figure 18b shows the contour of the surface which varied the initial velocity condition related to the force. The best configuration to harvest energy with a big interval of the amplitude is when the initial condition of the velocity of the horizontal coordinate is $x_{02}=0.2273$.

It is important to say that the dependence of the initial condition is only to the variation of the amplitude of the external force. Hence, the next numerical simulations will be performed considering $x_{02}=0.2273$. Next, the analysis of the amplitude of the external force is performed related to the average harvested power.

Figure 19 shows an analysis of the amplitude related to the average harvested power, a dimensional analysis (Fig. 19a) and a dimensionless analysis (Fig. 19b), and we see, as expected, that the higher the force amplitude, the more energy will be harvested. Because of the sensibility of the parameters, the acceptable dimensional range of values of the amplitude to this system is from $F_{0} \approx 0$ to
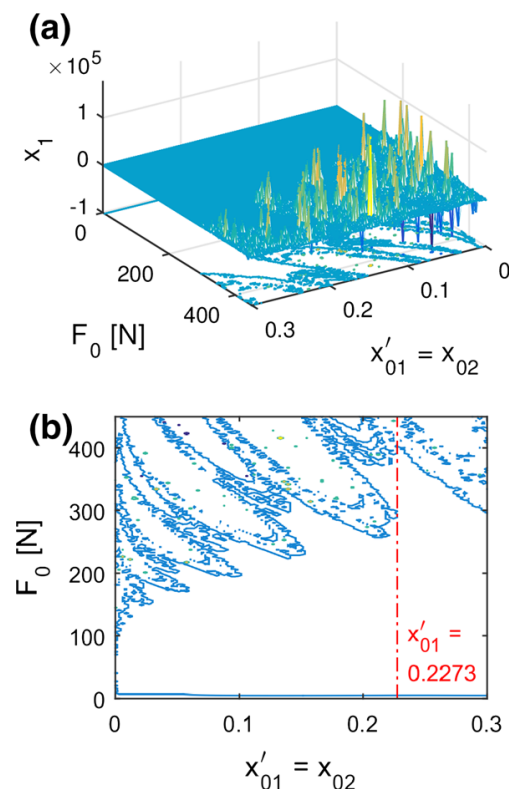

Fig. 18 Variation of the initial velocity of the horizontal movement and the amplitude of the external force related to the maximum displacement of the horizontal movement. a Surface. b Contour of the surface. $\omega_{n}=148 \mathrm{rad} / \mathrm{s}, \theta=0.3, \Theta=0$ 

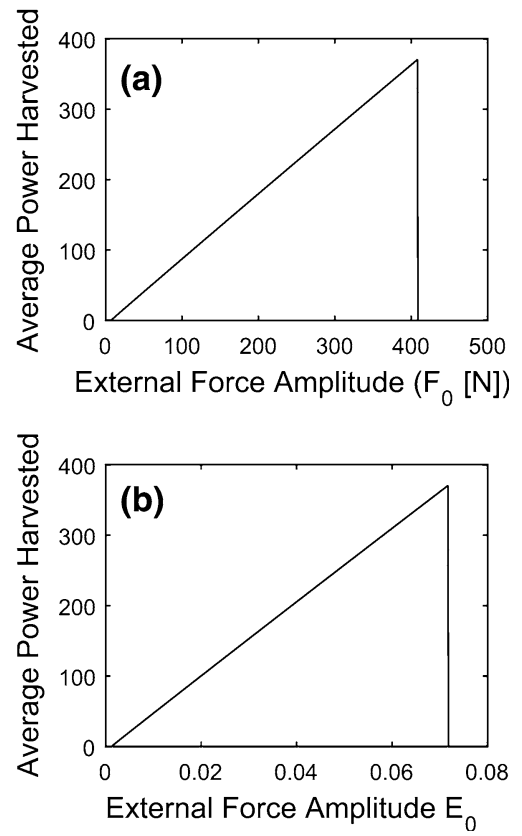

Fig. 19 External force amplitude analysis. a Dimensional analysis. b Dimensionless analysis, $\omega_{n}=148 \mathrm{rad} / \mathrm{s}, \theta=0.3$, $\Theta=0$

$F_{0} \approx 408.4 \mathrm{~N}$. After that, the system gets unstable. The same dimensional range to the dimensionless analysis is from $E_{0} \approx 0$ to $E_{0} \approx 0.0717$. In relation to the harvested power, the higher the external force amplitude the higher will be the harvested power.

In a few words, Table 3 shows some values of the average power related to the external force amplitude, showing its influence on the energy harvesting with only the linear piezoelectric coefficient. The amplitude will be varied from 10 to 400 because, with values of the amplitude higher than approximately 400, the system becomes unstable. Hence, we see that the higher the amplitude of the external force the higher will be the maximum horizontal displacement at steady state, the less will be the maximum vertical displacement and the higher will be the average harvested power, except at higher amplitudes as we see to $F_{0}>150 \mathrm{~N}$, approximately, that is when the vertical displacement begins to increase.

It is worth to study different combination of parameters with the amplitude of the external force. One of them is the piezoelectric coupling, because of its directly influence on the energy harvesting. Therefore, the next subsection discussed the combination of the amplitude and piezoelectric parameters.

\subsection{Linear piezoelectric coupling versus amplitude}

The interesting to combine the amplitude with the piezoelectric coefficient is that both parameters are strongly linked to the energy harvesting. Therefore, the influence of these two parameters on energy harvesting was validated.

The surface and its coloured contour shown in Fig. 20a, b, respectively, present the influence of the external force amplitude with the linear piezoelectric coupling related to the harvested power, varying the amplitude in the interval $0 \leq E_{0} \leq 0.07$ and the linear piezoelectric coefficient $0 \leq \theta \leq 0.35$. We observe that higher the amplitude higher will be the average power. However, to low amplitudes we can observe some decreases of power with the increasing of the linear piezoelectric coefficient as to $E_{0}=0.005$.

In addition, there are many values that the behaviour is unstable. It is possible to see in Fig. 20c, which shows the contour of the variation of the amplitude and linear piezoelectric parameters related to the maximum horizontal displacement of the system. The green and white spaces in the contour represent the unstable behaviour. The unique value of

Table 3 Effect of external force amplitude $\left(\omega_{n}=148 \mathrm{rad} / \mathrm{s}, \theta=0.3, \Theta=0\right)$

\begin{tabular}{lllll}
\hline Ext. force amplitude $F_{0}(\mathrm{~N})$ & Ext. force amplitude $E_{0}$ & Max. hor. disp. & Max. ver. disp. & Average power \\
\hline 10 & 0.0018 & 0.0267 & 0.0268 & 3.0253 \\
40 & 0.0070 & 0.0851 & 0.0261 & 31.3688 \\
120 & 0.0211 & 0.1563 & 0.0253 & 105.8645 \\
236.4 & 0.0415 & 0.2228 & 0.0263 & 213.2714 \\
250 & 0.0439 & 0.2294 & 0.0266 & 225.7571 \\
400 & 0.0702 & 0.2933 & 0.0305 & 362.7876 \\
\hline
\end{tabular}



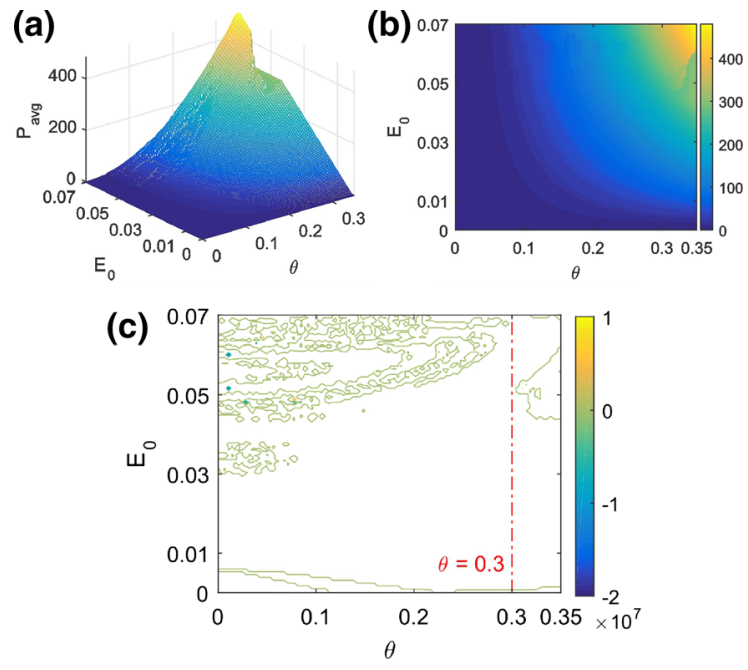

Fig. 20 Analysis of the amplitude of the external force versus linear piezoelectric coefficient versus average power neglecting the nonlinear piezoelectric coefficient. a Surface. b Contour of the surface; $\mathbf{c}$ analysis of the maximum displacement of the horizontal movement; $\omega_{n}=148 \mathrm{rad} / \mathrm{s}, \Theta=0$

the linear piezoelectric coefficient that there is no instability along the $0 \leq E_{0} \leq 0.07$ amplitude's interval is when $\theta=0.3$ (red line in Fig. 20c), the default linear piezoelectric coefficient used in this work.

Besides, the piezoelectric material contains a nonlinear contribution, which is very important to consider. In next subsection, we will show the influence of the nonlinear part to the combination of the amplitude and piezoelectric coupling.

\subsection{Linear and nonlinear piezoelectric coefficient versus amplitude}

The contribution of the nonlinear piezoelectric coefficient is very important because it approximates to the experimental curve of piezoelectric strain. Hence, the next numerical simulations will show the nonlinear contribution of the piezoelectric coefficient, considering its value fixed to $\Theta=1$. Now we will observe the most real effect of the piezoelectric material with the increasing of the amplitude of the external force.

Figure $21 \mathrm{a}, \mathrm{b}$ show a surface and a contour, respectively, of the analysis of the amplitudes interval $0 \leq F_{0} \leq 400 \mathrm{~N}\left(0 \leq E_{0} \leq 0.07\right)$ with the initial velocity conditions interval $0 \leq x_{02} \leq 0.3$. We observe a better description of the stable and unstable area. The whole white space is when the system is stable. The peaks in the surface, Fig. 21a, represents the
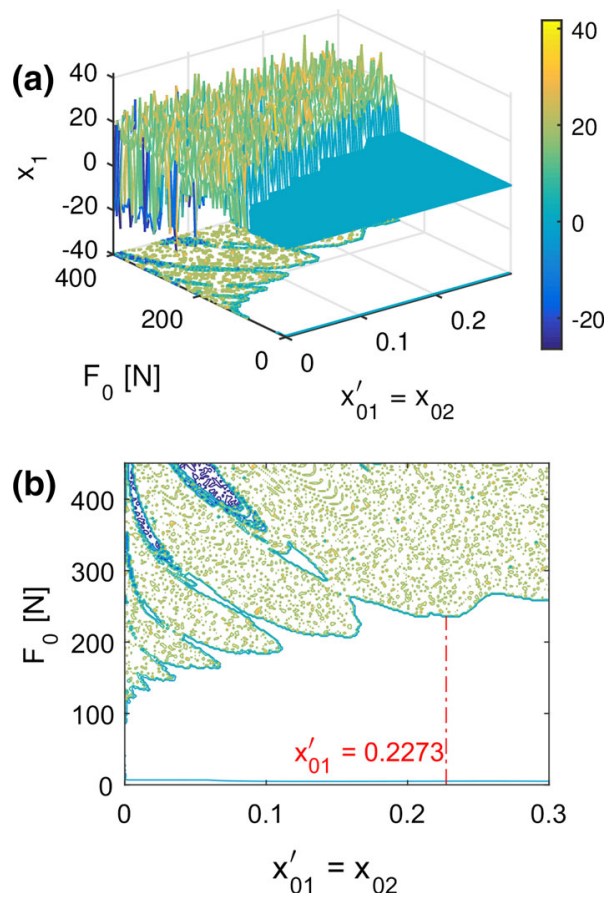

Fig. 21 Variation of the initial velocity of the horizontal movement and the amplitude of the external force related to the maximum displacement of the horizontal movement. a Surface. b Contour of the surface. $\omega_{n}=148 \mathrm{rad} / \mathrm{s}, \theta=0.3, \Theta=1$

unstable behaviour, while the blue area represents the stable behaviour of the system. The whole colored space, which are in the delimited contour area, Fig. $21 \mathrm{~b}$, is unstable. The interesting is that the initial condition of Sect. 5.1, which is $x_{02}=0.2273$, and has a stable behaviour varying the amplitude $0 \leq F_{0} \leq 400 \mathrm{~N}$, approximately, considering now the nonlinear piezoelectric coefficient $\Theta=1$, the amplitude varies in a smaller interval, that is the red dotted line in Fig. 21b.

Therefore, after the analysis of the stability considering the nonlinear contribution of the piezoelectric material, we will consider the same initial condition $x_{02}=0.2273$, and linear piezoelectric coefficient $\theta=$ 0.3 to the next simulations, in order to compare the behaviour of the system and the harvested power considering only the case of the sole linear piezoelectric coefficient (Sect. 5.1) and now considering the nonlinear piezoelectric coefficient $\Theta=1$.

Firstly, the analysis of the amplitude of the external force related to the harvested power is performed in Fig. 22. The stable interval of the dimensional and dimensionless amplitude is $0 \leq F_{0} \leq 236.4 \mathrm{~N}$ 

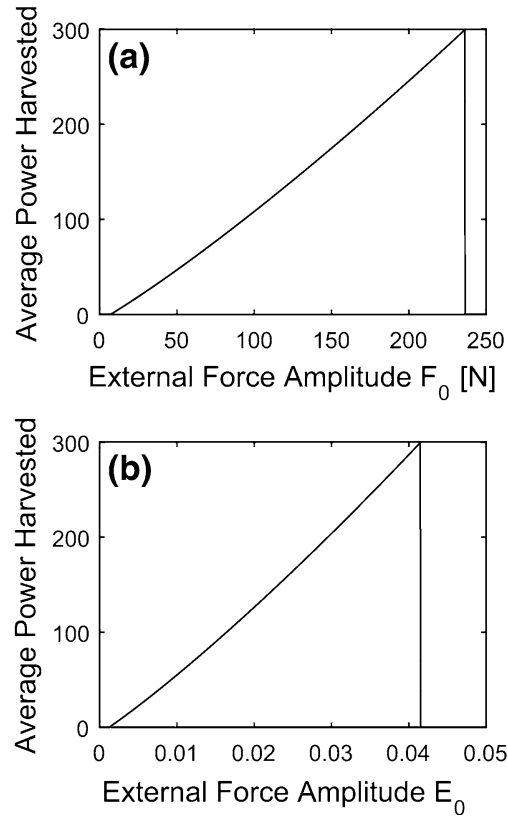

Fig. 22 External force amplitude analysis. a Dimensional analysis. b Dimensionless analysis, $\omega_{n}=148 \mathrm{rad} / \mathrm{s}, \theta=0.3$, $\Theta=1$

(Fig. 22a) and $0 \leq E_{0} \leq 0.0415$ (Fig. 22b), respectively. The harvested power has a linear gain with the increase of the amplitude, and the maximum amount of harvested power is 299.57 .

An overview of the influence of the nonlinear piezoelectric coefficient $\Theta=1$, in the variation of the external force amplitude is in Table 4 . We observe that, with the nonlinear piezoelectric coefficient, the vertical displacement always increase, as the horizontal displacement, and as the average harvested power.

A good way to see the influence of the nonlinear piezoelectric coefficient is comparing Table 3 with Table 4, carrying out some conclusions about the energy harvesting. Table 5 shows this comparison of the harvested power obtained with the sole linear case (fixed $\theta=0.3, \Theta=0)$ to the nonlinear case $(\Theta=1)$. The comparison $n^{\circ} 1$ is related to low amplitudes of the external force. When considering the case of the sole linear piezoelectric coefficient the harvested power is 3.0253 and considering both linear and nonlinear piezoelectric coefficient the harvested power is 2.9502, so we have loss of power. To high amplitudes we have gain of power as we can see at the comparisons $n^{\circ} 2-5$. The most important is, the gain of power at the highest amplitudes is very big. The system can provide more than 80 of gain of power, approximately.

Lastly, it was performed an analysis of the linear piezoelectric coefficient versus the amplitude of external force. It is important to see, in general, the stability and the optimum harvested power of the system, still considering the nonlinear contribution fixed in $\Theta=1$.

Figure 23 show a surface and their coloured contours related to the analysis of the external force versus linear piezoelectric coefficient related to the average power and the maximum displacement of the horizontal motion, both considering the effect of the nonlinear piezoelectric coefficient fixed in $\Theta=1$. From the surface and its coloured contour in Fig. 23a, $\mathrm{b}$, respectively, the average power keeps increasing with the increase of the amplitude and linear piezoelectric coefficient. However, the peak of power is not when the two parameters are the highest. The contour of Fig. 23c shows the regions of stability of the system. The behaviour of the system is unstable in all the regions in the delimited area. Nevertheless, inside the white space out of the delimited area is stable. The best configuration of stable behaviour and energy harvesting is between the red lined area. This area is described in Table 6.

Table 6 shows the best configuration of a stable behaviour of the system. The amplitude of the external

Table 4 Effect of external force amplitude $\left(\omega_{n}=148 \mathrm{rad} / \mathrm{s}, \theta=0.3, \Theta=1\right)$

\begin{tabular}{lllll}
\hline Ext. force amplitude $F_{0}(\mathrm{~N})$ & Ext. force amplitude $E_{0}$ & Max. hor. disp. & Max. ver. disp. & Average power \\
\hline 10 & 0.0018 & 0.0259 & 0.0259 & 2.9502 \\
40 & 0.0070 & 0.0846 & 0.0846 & 35.3240 \\
120 & 0.0211 & 0.1564 & 0.1564 & 134.2954 \\
180 & 0.0316 & 0.1943 & 0.1943 & 216.7544 \\
236.4 & 0.0415 & 0.2251 & 0.2251 & 299.5666 \\
\hline
\end{tabular}


Table 5 Comparison of harvested power neglecting and considering the nonlinear piezoelectric coefficient. Considering linear $\left.\theta=0.3, \omega_{n}=148 \mathrm{rad} / \mathrm{s}\right)$

\begin{tabular}{|c|c|c|c|c|c|c|}
\hline $\mathrm{N}^{\circ}$ & $\begin{array}{l}\text { Amplitude of the } \\
\text { external force } F_{0}(\mathrm{~N})\end{array}$ & $\begin{array}{l}\text { Amplitude of the } \\
\text { external force } E_{0}\end{array}$ & $\begin{array}{l}\text { Nonlinear piezoelectric } \\
\text { coefficient } \Theta\end{array}$ & Average power & $\%$ & Gain/loss \\
\hline \multirow[t]{2}{*}{1} & 10 & 0.0018 & 0 & 3.0253 & \multirow[t]{2}{*}{-2.48} & \multirow[t]{2}{*}{$\downarrow-$} \\
\hline & 10 & 0.0018 & 1 & 2.9502 & & \\
\hline \multirow[t]{2}{*}{2} & 40 & 0.0070 & 0 & 31.3688 & \multirow[t]{2}{*}{+12.61} & \multirow[t]{2}{*}{$\uparrow+$} \\
\hline & 40 & 0.0070 & 1 & 35.3240 & & \\
\hline \multirow[t]{2}{*}{3} & 120 & 0.0211 & 0 & 105.8645 & \multirow[t]{2}{*}{+26.86} & \multirow[t]{2}{*}{$\uparrow+$} \\
\hline & 120 & 0.0211 & 1 & 134.2954 & & \\
\hline \multirow[t]{2}{*}{4} & 180 & 0.0316 & 0 & 161.3566 & \multirow[t]{2}{*}{+34.33} & \multirow[t]{2}{*}{$\uparrow+$} \\
\hline & 180 & 0.0316 & 1 & 216.7544 & & \\
\hline \multirow[t]{2}{*}{5} & 236.4 & 0.0415 & 0 & 213.2714 & \multirow[t]{2}{*}{+40.46} & \multirow[t]{2}{*}{$\uparrow+$} \\
\hline & 236.4 & 0.0415 & 1 & 299.5666 & & \\
\hline
\end{tabular}
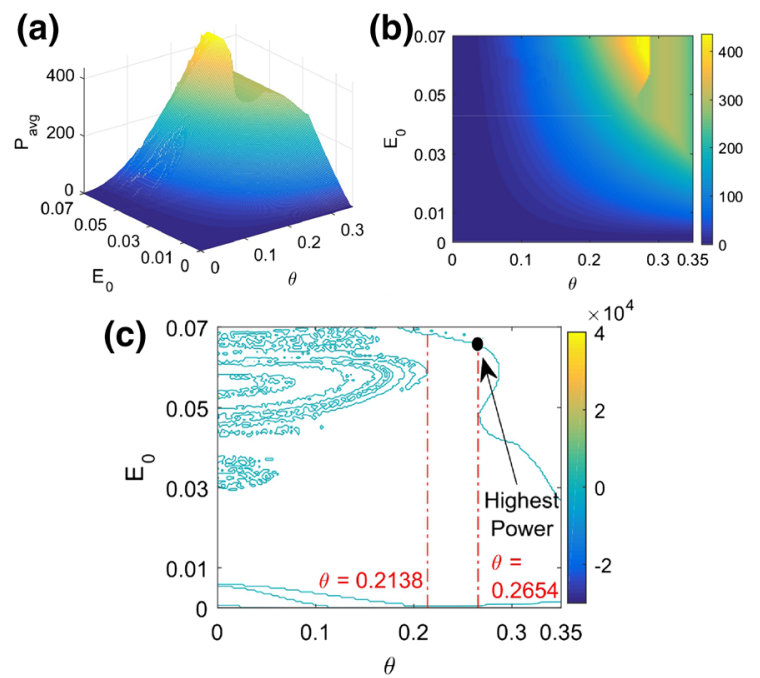

Fig. 23 Analysis of the amplitude of the external force versus linear piezoelectric coefficient versus average power neglecting the nonlinear piezoelectric coefficient. a Surface. b Contour of the surface; c analysis of the maximum displacement of the horizontal movement; $\omega_{n}=148 \mathrm{rad} / \mathrm{s}, \Theta=1$

force and linear piezoelectric coefficient can be linearly varied from zero (0) to the values of the Table 6 that are the two red lines of the contour in Fig. 23b. Between these two red lines, there is an area of other values that are stable too. The behaviour of this area is all periodic. Related to the average power, the maximum amount of power provided is when we have the highest values of the amplitude combined to the linear piezoelectric coefficient. In this case, is when $E_{0}=0.0653, \theta=0.2654$, that power is 404.0131.
Table 6 Best stable configuration of amplitude versus linear piezoelectric coefficient (with $\Theta=1$ )

\begin{tabular}{lll}
\hline$E_{0}\left(F_{0}(\mathrm{~N})\right)$ & $\theta$ & Average power \\
\hline $0.06812(387.9510 \mathrm{~N})$ & 0.2138 & 276.7696 \\
$0.0653(371.8908 \mathrm{~N})$ & 0.2654 & 404.0131 \\
\hline
\end{tabular}

We see that, with the nonlinear piezoelectric coefficient, the system has a greater area to be worked with stable and periodic behaviour than neglecting the nonlinear coefficient. While the highest amount of power of the sole linear case is 362.7876 with $E_{0}=0.0717$ and $\theta=0.3$, in the nonlinear case the highest amount of power is 404.0131 with a smaller value of amplitude and linear piezoelectric coefficient, which corresponds to the last line of Table 6.

\section{Conclusions}

In this work, we showed the saturation phenomenon, vibration energy transfer and the energy harvesting for a simple portal frame structure considering a nonlinear piezoelectric contribution. As the system is sensible to its parameters, extensive numerical analysis was presented. In addition, we showed the importance of the saturation on the improvement of the energy harvested in the system.

Still in the first section, we analyzed the energy transfer phenomenon in different ways. Firstly we 
considered just the two modes of vibration of the structure, then the piezoelectric material coupled to the columns. We could see the energy transfer phenomenon when there is saturation phenomenon, and also there is energy transferred to the piezoelectric coupling.

The frequency of the external force is a fundamental factor in order to the saturation phenomenon occurs and to the system keeps periodic. We showed a range of frequency values that the saturation occurred and some of those values can improve the energy harvesting. Table 7 represents a resume of the external force frequency topic.

Following the frequency analysis, the piezoelectric analysis was performed in order to harvest energy from the vibration of the columns. We observed that it was obtained great values and gains of power. The behaviour of the system kept periodic and it was able to harvest energy.

After, nonlinear piezoelectric coefficient was considered, fixed at $\Theta=1$, in order to see its influence to the energy harvesting. To the case of the frequency analysis, it was observed gains and losses of power, it will depend on the linear piezoelectric coefficient. Table 8 shows values of the linear piezoelectric coupling combining to the nonlinear piezoelectric coupling, resuming the entire frequency analysis topic.

In the section of the amplitude of the external force analysis, it was an extended section because its importance for the energy harvesting. As expected, the higher the amplitude, the higher the energy harvesting. However, the system can easily become unstable depending on the initial conditions. With different initial conditions, there was a different one which the system could use a great range of amplitude without become unstable, that was $x_{02}=0.2273$. The new initial condition provided an improvement to the energy harvesting because the system was opened to high values of the amplitude as we showed in the topic. Although, varying the linear piezoelectric coefficient, we could see that at low amplitudes, higher the linear coefficient, lower the harvested power.

After that, the analysis of the amplitude considering the nonlinear piezoelectric contribution was performed in order to see its influence in the energy harvesting. The same analysis of stability was performed too, showing that there was a greater gamma of variation of amplitude with linear piezoelectric coefficient related to the average power. Also, we could see the influence of the nonlinear coefficient to the amount of harvested power. We observed a great improvement

Table 7 Resume of external force frequency topic

\begin{tabular}{lll}
\hline Saturation phenomenon & External force frequency & Average harvested power \\
\hline$-\left(2 \omega_{1}=\omega_{2} \neq \Omega\right)$ & $100 \mathrm{rad} / \mathrm{s}(\Omega=1.1315)$ & $7.7702 \times 10^{-07}$ \\
$+\left(2 \omega_{1}=\omega_{2}=\Omega\right)$ & $148 \mathrm{rad} / \mathrm{s}(\Omega=2.0)$ & 31.3688 \\
$+\left(2 \omega_{1}=\omega_{2} \approx \Omega+\sigma\right)$ & $152 \mathrm{rad} / \mathrm{s}(\Omega=2.044)$ & 38.7119 \\
\hline
\end{tabular}

Table 8 Resume of the piezoelectric couplings topic

\begin{tabular}{llll}
\hline Linear coupling $\theta$ & Nonlinear coupling $\Theta$ & Average harvested power & Gain/loss \\
\hline 0.001 & 0 & 0.0024 & $\uparrow+$ \\
& 1 & 0.0029 & $\uparrow+$ \\
0.3 & 0 & 31.3688 & $\downarrow-$ \\
0.409 & 1 & 35.3240 & $\downarrow-$ \\
0.423 (max. linear stable) & 0 & 49.677 & $\downarrow$ \\
& 1 & 48.953 & \\
& 1 & 50.7971 & 10.7774 \\
\hline
\end{tabular}


Table 9 Resume of the amplitude of the external force topic considering $x_{02}=0.2273$

\begin{tabular}{lll}
\hline Piezoelectric case & Interval $F_{0}(\mathrm{~N}) / E_{0}$ & Average power \\
\hline Linear & $0 \leq F_{0} \leq 400 \mathrm{~N}$ & $0 \leq P_{\text {avg }} \leq 362.79$ \\
$\theta=0.3, \Theta=0$ & $0 \leq E_{0} \leq 0.07$ & \\
Nonlinear & $0 \leq F_{0} \leq 236.4 \mathrm{~N}$ & $0 \leq P_{\text {avg }} \leq 299.57$ \\
$\theta=0.3, \Theta=1$ & $0 \leq E_{0} \leq 0.0415$ & \\
Best case of nonlinear & $F_{0}=371.8908 \mathrm{~N}$ & 404.0131 \\
$\theta=0.0 .2654, \Theta=1$ & $E_{0}=0.0653$ & \\
\hline
\end{tabular}

of the harvested power from the system. In general, the resume of the last section is in Table 9.

Acknowledgements The authors acknowledge support by CNPq (Grant: 447539/2014-0), CAPES and FAPESP, all Brazilian research funding agencies.

\section{References}

1. Erturk A, Hoffmann J, Inman DJ (2009) A piezomagnetoelastic structure for broadband vibration energy harvesting. Appl Phys Lett 94(25):254102. doi:10.1063/1.3159815

2. Litak G, Friswell MI, Kwuimy CAK, Adhikari S, Borowiec M (2012) Energy harvesting by two magnetopiezoelastic oscillators with mistuning. Theor Appl Mech Lett 2(4):043009. doi:10.1063/2.1204309

3. Stanton SC, McGehee CC, Mann BP (2010) Nonlinear dynamics for broadband energy harvesting: investigation of a bistable piezoelectric inertial generator. Phys D Nonlinear Phenom 239(10):640-653. doi:10.1016/j.physd.2010.01.019

4. Syta A, Bowen CR, Kim HA, Rysak A, Litak G (2015) Experimental analysis of the dynamical response of energy harvesting devices based on bistable laminated plates. Meccanica 50(8):1961-1970. doi:10.1007/s11012-0150140-1

5. Friswell MI, Bilgen O, Ali SF, Litak G, Adhikari S (2015) The effect of noise on the response of a vertical cantilever beam energy harvester. ZAMM - J Appl Math Mech 95(5):433-443. doi:10.1002/zamm.201300183

6. Litak G, Friswell MI, Adhikari S (2015) Regular and chaotic vibration in a piezoelectric energy harvester. Meccanica 51:1017-1025. doi:10.1007/s11012-015-0287-9

7. Erturk A, Inman DJ (2011) Piezoelectric energy harvesting from aeroelastic vibrations. In: Piezoelectric energy harvesting. Wiley, Chichester. doi:10.1002/9781119991151

8. Priya S, Inman DJ (eds) (2009) Energy harvesting technologies, vol 21. Springer, New York. doi:10.1007/978-0387-76464-1

9. Stephen NG (2006) On energy harvesting from ambient vibration. J Sound Vib 293(1):409-425. doi:10.1016/j.jsv. 2005.10.003

10. Jalili N (2010) Piezoelectric-based vibration control: from macro to micro/nano scale systems. Springer, US. doi:10. 1007/978-1-4419-0070-8

11. Preumont A (2006) Mechatronics: dynamics of electromechanical and piezoelectric systems, vol 136. Springer, Netherlands. doi:10.1007/1-4020-4696-0
12. DuToit NE, Wardle BL (2007) Experimental verification of models for microfabricated piezoelectric vibration energy harvesters. AIAA J 45(5):1126-1137. doi:10.2514/ 1.25047

13. Twiefel J, Richter B, Sattel T, Wallaschek J (2008) Power output estimation and experimental validation for piezoelectric energy harvesting systems. J Electroceram 20(3-4):203-208. doi:10.1007/s10832-007-9168-5

14. Crawley EF, Anderson EH (1990) Detailed models of piezoceramic actuation of beams. J Intell Mater Syst Struct 1(1):4-25. doi:10.2514/6.1989-1388

15. Triplett A, Quinn DD (2009) The effect of non-linear piezoelectric coupling on vibration-based energy harvesting. J Intell Mater Syst Struct 20(16):1959-1967. doi:10. 1177/1045389X09343218

16. Daqaq MF, Masana R, Erturk A, Quinn DD (2014) On the role of nonlinearities in vibratory energy harvesting: a critical review and discussion. Appl Mech Rev 66(4):040801. doi:10.1115/1.4026278

17. Iliuk I, Balthazar JM, Tusset AM, Piqueira JRC, de Pontes BR, Felix JLP, Bueno M (2013) A non-ideal portal frame energy harvester controlled using a pendulum. Eur Phys J 222(7):1575-1586 (special topics). doi:10.1140/epjst/ e2013-01946-4

18. Iliuk I, Balthazar JM, Tusset AM, Piqueira JR, de Pontes BR, Felix JL, Bueno M (2013) Application of passive control to energy harvester efficiency using a nonideal portal frame structural support system. J Intell Mater Syst Struct 25(4). doi:10.1177/1045389X13500570

19. Balthazar JM, Rocha RT, Brasil RMFL, Tusset AM, de Pontes BR, Silveira M (2014) Mode saturation, mode coupling and energy harvesting from ambient vibration in a portal frame structure. In: ASME 2014 international design engineering technical conferences and computers and information in engineering conference (pp V008T11A044V008T11A044). American Society of Mechanical Engineers. doi:10.1115/DETC2014-34268

20. Iliuk I, Brasil RMLRF, Balthazar JM, Tusset AM, Piccirillo V, Piqueira JRC (2014) Potential application in energy harvesting of intermodal energy exchange in a frame: FEM analysis. Int J Struct Stab Dynamics 14(8). doi:10.1142/ S0219455414400276

21. Nayfeh AH, Mook DT, Marshall LR (1973) Nonlinear coupling of pitch and roll modes in ship motions. J Hydronaut 7(4):145-152. doi:10.2514/3.62949

22. Nayfeh AH, Mook DT (2008) Nonlinear oscillations. Wiley, United States, p 720

23. Mook DT, Plaut RH, HaQuang N (1985) The influence of an internal resonance on non-linear structural vibrations under 
subharmonic resonance conditions. J Sound Vib 102(4):473-492. doi:10.1016/S0022-460X(85)80108-5

24. Nayfeh AH (2000) Nonlinear interactions: analytical, computational and experimental methods. In: Nonlinear Science. Wiley, New York, p 760

25. Mankala R, Quinn DD (2004) Resonant dynamics and saturation in a coupled system with quadratic nonlinearities. In: Proceedings of the ASME-DETC conference, anaheim, 621-626. doi:10.1115/IMECE2004-61623

26. Quinn DD (2007) Resonant dynamics in strongly nonlinear systems. Nonlinear Dyn 49(3):361-373. doi:10.1007/ s11071-006-9126-9

27. Golnaraghi MF (1991) Vibration suppression of flexible structures using internal resonance. Mech Res Commun 18(2-3):135-143. doi:10.1016/0093-6413(91)90042-U

28. Oueini SS, Nayfeh AH, Golnaraghi MF (1997) A theoretical and experimental implementation of a control method based on saturation. Nonlinear Dyn 13(2):189-202. doi:10.1023/ A:1008207124935

29. Oueini SS (1999) Techniques for controlling structural vibrations, PhD Thesis, Virginia Tech US

30. Pai PF, Schulz MJ (2000) A refined nonlinear vibration absorber. Int J Mech Sci 42(3):537-560. doi:10.1016/ S0020-7403(98)00135-0

31. Pai PF, Wen B, Naser AS, Schulz MJ (1998) Structural vibration control using PZT patches and non-linear phenomena. J Sound Vib 215(2):273-296. doi:10.1006/jsvi. 1998.1612

32. Shoeybi M, Ghorashi M (2005) Control of a nonlinear system using the saturation phenomenon. Nonlinear Dyn 42(2):113-136. doi:10.1007/s11071-005-4123-y

33. Warminski J, Cartmell MP, Mitura A, Bochenski M (2013) Active vibration control of a nonlinear beam with self-and external excitations. Shock Vib 20(6):1033-1047. doi:10. 3233/SAV-130821

34. Felix JLP, Balthazar JM, Brasil RM (2005) On saturation control of a non-ideal vibrating portal frame foundation type shear-building. J Vib Control 11(1):121-136. doi:10.1177/ 1077546305047656

35. Tusset AM, Piccirillo V, Bueno AM, Balthazar JM, Sado D, Felix JLP, da Fonseca RMLR (2015) Chaos control and sensitivity analysis of a double pendulum arm excited by an RLC circuit based nonlinear shaker. J Vib Control 22(17):3621-3637. doi:10.1177/1077546314564782

36. Felix JLP, Silva EL, Balthazar JM, Tusset AM, Bueno AM, Brasil RMLRF (2014) On nonlinear dynamics and control of a robotic arm with chaos. In: MATEC Web of Conferences (Vol 16, p 05002). EDP Sciences. doi:10.1051/ matecconf/20141605002

37. Hall BD, Mook DT, Nayfeh AH, Preidikman S (2001) Novel strategy for suppressing the flutter oscillations of aircraft wings. AIAA J 39(10):1843-1850. doi:10.2514/2. 1190

38. Pratt JR, Oueini SS, Nayfeh AH (1999) Terfenol-D nonlinear vibration absorber. J Intell Mater Syst Struct 10(1):29-35. doi:10.1177/1045389X9901000104

39. Ashour ON, Nayfeh AH (2002) Adaptive control of flexible structures using a nonlinear vibration absorber. Nonlinear Dyn 28(3-4):309-322. doi:10.1023/A:1015622630382

40. Balthazar JM, Felix JLP, Brasil RMLRD (2003) On nonlinear dynamics and control of a particular portal frame foundation model, excited by a non-ideal motor. Mater Sci Forum 440:371-380. doi:10.4028/www.scientific.net/MSF. 440-441.371

41. Balthazar JM, Felix JL, Brasil RM, Krasnopolskaya TS, Shvets AY (2009) Nonlinear interactions in a piezoceramic bar transducer powered by a vacuum tube generated by a nonideal source. J Comput Nonlinear Dyn 4(1):011013. doi:10.1115/1.3007909

42. Vakakis AF (2008) Nonlinear targeted energy transfer in mechanical and structural systems, vol 156. Springer, Netherlands. doi:10.1007/978-1-4020-9130-8

43. Tusset AM, Balthazar JM, Chavarette FR, Felix JLP (2012) On energy transfer phenomena, in a nonlinear ideal and nonideal essential vibrating systems, coupled to a (MR) magneto-rheological damper. Nonlinear Dyn 69(4): 1859-1880. doi:10.1007/s11071-012-0391-5

44. Tusset AM, Balthazar JM, Felix JLP (2012) On elimination of chaotic behavior in a non-ideal portal frame structural system, using both passive and active controls. J Vib Control 19(6):803-813. doi:10.1177/1077546311435518 